\title{
Tumor-infiltrating CD8+ lymphocytes predict different clinical outcomes in organ and non-organ-confined urothelial carcinoma of the bladder following radical cystectomy
}

\author{
Shiqiang Zhang ${ }^{\text {Corresp., }}{ }^{1,2}$ ， Jun Wang ${ }^{1}$, Xinyu Zhang ${ }^{3}$ ， Fangjian Zhou ${ }^{\text {Corresp. }}{ }^{2}$ \\ 1 Zhongshan School of Medicine, Sun Yat-sen University, Guangzhou, China \\ 2 Department of Urology, Sun Yat-sen University Cancer Center, Guangzhou, China \\ ${ }^{3}$ Shenzhen Luohu Maternity and Child Healthcare Hospitala, Shenzhen, Chin \\ Corresponding Authors: Shiqiang Zhang, Fangjian Zhou \\ Email address: zhangshq9@mail2.sysu.edu.cn, zhoufj@sysucc.org.cn
}

Tumor-infiltrating lymphocytes (TILs) are associated with better clinical outcomes in many tumors. TILs represent a cell-mediated immune response against the carcinoma. CD8+ TILs are a crucial component of cell-mediated immunity. The significance of CD8+ TILs has not been reported respectively in organ and non-organ-confined urothelial carcinoma (UC) of the bladder. We explored the prognostic value of CD8+ TILs in the two groups. The presence of CD8+ TILs was assessed by immunohistochemical staining of whole tissue sections from 75 organ and 51 non-organ-confined disease patients with long-term followup, and its correlation with clinicopathological features and overall survival (OS) was determined. The CD8+ TIL immunohistochemical staining score was $0(<1 \%), 1(\geq 1 \%), 2$ $(\geq 5 \%)$, or $3(\geq 10 \%)$ based on the percentage of positively stained cells out of total cells. $A$ patient was considered CD8 negative if the score was 0 . There were no associations between CD8+ TILs and age, sex, nuclear grade, and adjuvant or neoadjuvant chemotherapy in organ and non-organ-confined disease. The presence of CD8+ TILS was seen more frequently in $\mathrm{pTa}_{-}{ }_{1}$ than $\mathrm{pT}_{2}$ stage $(p=0.033)$ in organ-confined disease. No associations between CD8+ TILs and $\mathrm{pT}$ stage, $\mathrm{pN}$ stage were found in non-organ-confined disease. CD8+ TILs were associated with better OS (log-rank test, $P=0.036$ ) in non-organconfined disease, but with poorer OS (log-rank test, $\mathrm{P}=0.040$ ) in organ-confined disease by the Kaplan-Meier method. In multivariate analysis, CD8+ TILs were an independent favorable prognostic factor in non-organ-confined disease, but were an independent unfavorable prognostic factor in organ-confined disease. These results suggest that CD8+ TILs have clinically significant anti-tumor activity in non-organ-confined disease, but may have pro-tumor activity in organ-confined disease. Therefore, we should be cautious if CD8+ TILs are aimed to be exploited in the treatment of bladder cancer. 
1 Tumor-infiltrating CD8+ lymphocytes predict different clinical outcomes in organ and 2 non-organ-confined urothelial carcinoma of the bladder following radical cystectomy

3 Shiqiang Zhang ${ }^{1,2}$, Jun Wang ${ }^{1}$, Xinyu Zhang ${ }^{3}$, and Fangjian Zhou ${ }^{2}$

41 Zhongshan School of Medicine, Sun Yat-sen University, Guangzhou, China

52 Department of Urology, Sun Yat-sen University Cancer Center, Guangzhou, China

63 Shenzhen Luohu Maternity and Child Healthcare Hospital, Shenzhen, China

7

8 Corresponding authors: Shiqiang Zhang, zhangshq9@mail2.sysu.edu.cn; Fangjian Zhou, 9 zhoufj@sysucc.org.cn 
13

14

\section{Abstract}

Tumor-infiltrating lymphocytes (TILs) are associated with better clinical outcomes in many tumors. TILs represent a cell-mediated immune response against the carcinoma. CD8+ TILs are a crucial component of cell-mediated immunity. The significance of CD8+ TILs has not been reported respectively in organ and non-organ-confined urothelial carcinoma (UC) of the bladder. We explored the prognostic value of CD8+ TILs in the two groups. The presence of CD8+ TILs was assessed by immunohistochemical staining of whole tissue sections from 75 organ and 51 non-organ-confined disease patients with long-term follow-up, and its correlation with clinicopathological features and overall survival (OS) was determined. The CD8+ TIL immunohistochemical staining score was $0(<1 \%), 1(\geq 1 \%), 2(\geq 5 \%)$, or $3(\geq 10 \%)$ based on the percentage of positively stained cells out of total cells. A patient was considered CD8 negative if the score was 0 . There were no associations between CD8+ TILs and age, sex, nuclear grade, and adjuvant or neoadjuvant chemotherapy in organ and non-organ-confined disease. The presence of CD8+ TILs was seen more frequently in $\mathrm{pTa}_{-1}$ than $\mathrm{pT}_{2}$ stage $(\mathrm{p}=0.033)$ in organ-confined disease. No associations between CD8+ TILs and pT stage, pN stage were found in non-organconfined disease. CD8+ TILs were associated with better OS (log-rank test, $\mathrm{P}=0.036)$ in nonorgan-confined disease, but with poorer OS (log-rank test, $\mathrm{P}=0.040)$ in organ-confined disease by the Kaplan-Meier method. In multivariate analysis, CD8+ TILs were an independent favorable prognostic factor in non-organ-confined disease, but were an independent unfavorable prognostic factor in organ-confined disease. These results suggest that CD8+ TILs have clinically significant anti-tumor activity in non-organ-confined disease, but may have pro-tumor activity in organ-confined disease. Therefore, we should be cautious if CD8+ TILs are aimed to be exploited in the treatment of bladder cancer. 


\section{Introduction}

Bladder cancer is the ninth leading cause of cancer death in both sexes, with an estimated 429,800 new cases and 165,100 deaths in 2012 worldwide (Torre et al., 2015; Ferlay et al., 2015). In the US, bladder cancer was the second most common genitourinary cancer, with projected 79,030 new cases and 16,870 related deaths in 2017 (Siegel et al., 2017). In China, there were an estimated 80,500 new cases and 32,900 deaths from bladder cancer in 2015 (Chen et al., 2016).

Bladder cancer can be divided into organ-confined (pTa/pT1/carcinoma in situ [CIS]/pT2 NX/N0 M0) and non-organ-confined (pT3/T4N any M any or pT any N+M any or pT any N any $\mathrm{M}+$ ) disease at cystectomy based on extravesical invasion (Boorjian et al., 2008). More than 90\% of bladder cancers are urothelial carcinoma (UC) (Kaufman et al., 2009), of which one characteristic is the presence of high rates of somatic mutations (2014). These mutations may produce an increased number of cancer neoantigens and enhance the ability of the host immune system to recognize tumor cells (Chen \& Mellman, 2013; Sanmamed \& Chen, 2014; Chen, 2014).

The presence of tumor-infiltrating lymphocytes (TILs) has been associated with favorable prognosis in several neoplasms (Pages et al., 2005; Galon et al., 2006; Laghi et al., 2009; Zhang et al., 2003; Wang et al., 2016; Mahmoud et al., 2011). Among the TILs, CD8+ TILs may play a central role in anti-tumor immunity. Non-organ-confined UC of the bladder has worse prognosis than organ-confined disease. However, only two reported studies have assessed the effect of CD8+ TILs in UC of the bladder following radical cystectomy, which suggest the presence of CD8+ TILs is associated with favorable prognosis.. If CD8+ TILs have the similar role in the two group patients of organ and non-organ confined disease, there hasn't been reported.

In this study, we evaluated CD8+ TILs by immunohistochemistry (IHC) in organ and nonorgan-confined UC of the bladder following radical cystectomy, and explored the association with overall survival (OS). 
63

64

65

66

67

\section{Materials and Methods}

\section{Patients and tumor specimens}

Archival pathology tumor blocks were obtained from the Department of Pathology of Sun Yat-sen University Cancer Center, Guangzhou, China. We retrieved 126 consecutive formalinfixed, paraffin-embedded radical cystectomy specimens of UC of the bladder obtained between January 2000 and September 2009, including 51 non-organ-confined and 75 organ-confined disease. Representative tumor specimens were selected upon review of hematoxylin and eosin slides. We reviewed all sections to confirm the original diagnosis and staged them according to the 2010 American Joint Committee on Cancer TNM (tumor-node-metastasis) classification. The clinicopathological features were retrospectively reviewed from the patients' medical records. The OS was determined from the date of surgery to the date of death or censored on the date of the last follow-up. Written informed consent was obtained from all patients prior to the study. The Ethical Committee of Sun Yat-sen University Cancer Center approved all experimental methods in the study (YB2016-006).

\section{CD8 IHC}

8

CD8 IHC was conducted according to a standard method. Briefly, tissue sections were deparaffinized and rehydrated. Endogenous peroxidase activity was blocked with 3\% hydrogen peroxide for $15 \mathrm{~min}$. For antigen retrieval, tissue slides were boiled in $10 \mathrm{mM}$ citrate buffer $(\mathrm{pH}$ 6.0) in antigen retriever (Ascend Biotechnology, Guangzhou, China) at $120^{\circ} \mathrm{C}$ for $5 \mathrm{~min}$. Nonspecific binding was blocked with Serotec Block ACE (AbD Serotec, Oxford, UK) for 15 min. The slides were incubated with anti-CD8 antibody (Clone SP16, Thermo Scientific, Fremont, USA) overnight at $4^{\circ} \mathrm{C}$. All incubations were performed in a moist chamber. Subsequently, the slides were incubated with a horseradish peroxidase-labeled secondary antibody for $60 \mathrm{~min}$ at $37^{\circ} \mathrm{C}$, and then visualized using 3,3'-diaminobenzidine. 
87

88

89

90

91

92

93

94

95

96

97

106

107

108

109

110

\section{Scoring of CD8+ TILs}

8
scoring. The CD8+ TIL score was $0(<1 \%), 1(\geq 1 \%), 2(\geq 5 \%)$, or $3(\geq 10 \%)$ based on the estimated percentage of positively stained cells out of total cells. A patient with a score of 0 was considered CD8 negative (CD8-). If there was a discrepancy in scoring, both pathologists reviewed the case using a double-headed microscope to achieve consensus. About $31 \%$ of the total cases required a consensus discussion between the reviewing pathologists for scoring. At all times, the pathologists were blinded to the clinical outcome.

\section{Statistical methods}

The Fisher exact test was used to evaluate CD8 status between different categories. The Kaplan-Meier method and Cox proportional hazards model were used for OS analysis; differences in the Kaplan-Meier survival analysis were assessed using the log-rank test. Statistical analysis was performed using IBM SPSS 19.0 (SPSS, Inc., Chicago, Illinois) for Windows; the significance level was set at 0.05 (2-sided).

\section{Results}

Clinicopathological analysis of organ and organ-confined disease and presence of CD8+TILs

Table 1 summarizes the clinicopathologic characteristics. The median age of the 51 patients at surgical resection was 63 years, and the median follow-up time from date of surgical resection was 30.5 months (range: 0.2-129.6 months) in non-organ-confined disease. In organ-confined disease, median age of the 75 patients at surgical resection was 60 years, and median follow-up time from date of surgical resection was 51.8 months (range: 0.3-139.7 months).

CD8+ TILs were detected in both the tumoral and intratumoral areas, and were distributed mainly at the interface of the tumor and tumor-adjacent stroma (Fig.1). We compared CD8+ TILs with the clinicopathological characteristics of organ and non-organ-confined disease (Table 
111 1). There were no associations between CD8+ TILs and age, sex, nuclear grade, and adjuvant or 112 neoadjuvant chemotherapy in both organ and non-organ-confined disease. In organ-confined 113 disease, the presence of CD8 TILs was seen more frequently in $\mathrm{pTa}_{-1}$ than $\mathrm{pT}_{2}$ stage $(\mathrm{p}=0.033)$. 114 No associations between CD8+ TILs and pT stage, $\mathrm{pN}$ stage were found in non-organ-confined 115 disease.

\section{OS and Cox proportional hazards analysis}

118

119

120

121

122

123

124

125

126

127

128

129

130

131

132

133

134

135

136

We examined the relationship between the presence of CD8+ TILs and OS from the date of cystectomy. There was no statistically significant association between CD8+ TILs and OS in all $126 \mathrm{UC}$ of the bladder patients (OS, P =0.899, Fig. 2A) by the Kaplan-Meier method . The presence of CD8+ TILs was correlated with improved OS in 51 patients of non-organ-confined disease $(\mathrm{P}=0.036$, Fig. 2B). On the contrast, it was associated with poorer OS in 75 patients of organ-confined disease ( $\mathrm{P}=0.040$, Fig. $2 \mathrm{C})$. The multivariate analysis showed that the presence of CD8+ TILs was an independent prognostic predictor in both organ and non-organ-confined disease. In organ-confined disease, the patients with CD8 negative had a better prognosis than the patients with CD8 positive (RR: $0.212,95 \%$ CI: $0.048-0.932, \mathrm{P}=0.0040$; Table 2). However, in non-organ-confined disease, the patients with CD8 negative had a worse prognosis than the patients with CD8 positive (RR: 2,397, 95\% CI: 1.031-5.573, $\mathrm{P}=0.042$; Table 2).

In the present study, the CD8+ TIL score was $0(<1 \%), 1(\geq 1 \%), 2(\geq 5 \%)$, or $3(\geq 10 \%)$ based on the estimated percentage of positively stained cells out of total cells. It was no statistically significant association between CD8+ TILs and OS in all 126 UC of the bladder patients by the Kaplan-Meier method regardless of CD8 score $=1 \& 2 \& 3$ vs CD8 score $=0$, CD8 score $=0 \&$ 1 vs CD8 score $=2 \& 3$ ). There was no statistically significant association in non-organ-confined disease or pT3+4 stage (data not shown) when it was CD8 score $=0 \& 1$ vs CD8 score $=2 \& 3$; but when it was $\mathrm{CD} 8$ score $=1 \& 2 \& 3$ vs $\mathrm{CD} 8$ score $=0$, there was statistically significant association in non-organ-confined disease or pT3+4 stage (data not shown). 
138

139

140

141

142

143

144

145

146

147

148

149

150

151

152

153

154

155

156

157

158

159

160

161

162

\section{Discussion}

We show that CD8+ TILs are significantly associated with favorable clinical outcome in nonorgan-confined disease, but with unfavorable clinical outcome in organ-confined disease in the Kaplan-Meier survival analysis, although no statistically significant association between CD8+ TILs and OS was found in all 126 UC of bladder. In multivariate Cox's proportional hazards regression analysis, the presence of CD8+ TILs was an independent factor of prognosis in both of organ-confined and non-organ confined disease. It was a favorable factor of prognosis in non-organ-confined disease, but was an unfavorable factor in organ-confined disease.

Previous studies have shown that TILs (especially CD8+ TILs), which have anti-tumor activity, are associated with good prognosis in patients with tumors (Galon et al., 2006; Mahmoud et al., 2011; Hwang et al., 2012; Pages et al., 2005). CD8+ TILs mediate most antitumor immune responses (Wang et al., 2016), bladder cancer studies also suggest that the presence of CD8+ TILs is associated with favorable prognosis (Sharma et al., 2007; Faraj et al., 2015). High CD8 density was associated with improved OS $(\mathrm{P}=0.02)$ in a subset of 50 cystectomies for invasive bladder UC ( $\geq$ pT1) (Faraj et al., 2015). The authors defined high CD8 density as the presence of $\geq 60 \mathrm{CD} 8+T$ cells per high-power field in a given tissue microarray spot. A tumor was considered high density if $50 \%$ of its spots were of high density. This is different from our study. A patient with a score of $0(<1 \%$ of positively stained cells out of total cells ) was considered CD8 negative in our study. CD8+ T cells were detected in both the tumoral and intratumoral areas, and were distributed mainly at the interface of the tumor and tumor-adjacent stroma (Tumeh et al., 2014), the score of CD8+ TILs was accessed in tumor periphery ( i.e. the interface of the tumor and tumor-adjacent stroma ) in the present study. Another study showed that, in similar-staged bladder UC (pT2, pT3, or pT4), patients with more CD8+ TILs within the tumor had better OS $(\mathrm{P}<0.018)$ than patients with fewer CD8+ TILs (Sharma et al., 2007). Multivariate analysis also revealed a significant association between CD8+ 
163 TILs and OS. The previous two reports had the similar results, and it was consistent with the 164 findings of the present study in non-organ confined disease. However, the presence of CD8+ 165 TILs is an significant unfavorable predictor in organ-confined disease, which was also found in 166 the present study. The immune cells of CD8+ TILs have anti-tumor activity in non-organ 167 confined disease, and may have pro-tumor activity in organ-confined disease. But if the CD8+ 168 TILs themselves had pro-tumor activity or CD8+ TILs' anti-tumor activity inhibited by other 169 powerful pro-tumor factors in organ-confined disease, such as tumor cells PD-L1 expression 170 (Chen \& Flies, 2013), it needs further investigation. Many studies suggest CD8+ TILs could 171 potentially be exploited in the treatment of cancer. If CD8+ TILs are aimed to be exploited in the 172 treatment of bladder cancer, that different therapeutic strategies should be considered, which was 173 indicated by the present study.

174 The efficacy of immunotherapy in superficial bladder tumors was first established in 1976 175 with BCG (Morales et al., 1976), but no immunotherapy had been approved for the treatment of 176 advanced disease, until anti-PD-1/PD-L1 monoclonal antibody were approved for the treatment 177 of bladder cancer patients (Powles et al., 2017). All of the patients in our study didn't receive any 178 immunotherapy.

The study limitations are the small number of patients included, the heterogeneity of the 180 patient population and contrasting results between organ and non-organ-confined disease. Therefore, further investigation is necessary to verify our findings, and explore why the presence CD8+ TILs have different effects in the two cohorts.

In conclusion, our results provide evidence of the prognostic importance of CD8+ TILs in organ and non-organ-confined UC of the bladder. The presence of CD8+ TILs in non-organconfined disease correlates with improved OS, but it was correlated with poorer OS in organconfined disease. On this account, if CD8+ TILs are aimed to be exploited in the treatment of bladder cancer, different treatment strategies should be considered. 
188

189

190

191

192

193

194

195

196

197

198

199

200

201

202

203

204

205

206

207

208

209

210

211

212

213

214

215

216

217

218

219

220

\section{Acknowledgements}

We thank Prof. Lieping Chen (Yale University) for experimental design and laboratory assistance. We thank Xinke Zhang (Sun Yat-sen University Cancer Center) and Weisong Li (The First Affiliated Hospital of Sun Yat-sen University) for their assistance with the scoring of CD8+ TILs.

\section{Figure legends}

Figure 1. Representative examples of CD8+ TILs immunostaining. CD8+ TILs are distributed mainly at the interface of the tumor and tumor-adjacent stroma. A, B, C and D $(\times 100$ magnification) are representative images of scores of $0,1,2$, and 3, respectively.

Figure 2. Kaplan-Meier survival curves with log-rank testing. A. All UC of the bladder (CD8+, 90; CD8-, 36). B. Non-organ-confined UC of the bladder (CD8+, 35; CD8-, 16). C. Organconfined UC of the bladder (CD8+, 55; CD8-, 20).

\section{References:}

2014. Comprehensive molecular characterization of urothelial bladder carcinoma. Nature 507:315-322. 10.1038/nature12965

Boorjian, S.A., Sheinin, Y., Crispen, P.L., Farmer, S.A., Lohse, C.M., Kuntz, S.M., Leibovich, B.C., Kwon, E.D., and Frank, I. 2008. T-cell coregulatory molecule expression in urothelial cell carcinoma: clinicopathologic correlations and association with survival. Clinical Cancer Research 14:4800-4808. 10.1158/1078-0432.CCR-08-0731

Chen, D.S., and Mellman, I. 2013. Oncology meets immunology: the cancer-immunity cycle. Immunity 39:1-10. 10.1016/j.immuni.2013.07.012

Chen, L. 2014. From the guest editor: Tumor site immune modulation therapy. Cancer Journal 20:254-255. 10.1097/PPO.0000000000000062

Chen, L., and Flies, D.B. 2013. Molecular mechanisms of T cell co-stimulation and co-inhibition. Nature Reviews Immunology 13:227-242. 10.1038/nri3405

Chen, W., Zheng, R., Baade, P.D., Zhang, S., Zeng, H., Bray, F., Jemal, A., Yu, X.Q., and He, J. 2016. Cancer statistics in China, 2015. CA Cancer J Clin 66:115-132. 10.3322/caac.21338

Faraj, S.F., Munari, E., Guner, G., Taube, J., Anders, R., Hicks, J., Meeker, A., Schoenberg, M., Bivalacqua, T., Drake, C., and Netto, G.J. 2015. Assessment of tumoral PD-L1 expression and 
221 intratumoral CD8+ $\mathrm{T}$ cells in urothelial carcinoma. Urology 85:701-703.

222 10.1016/j.urology.2014.10.020

223 Ferlay, J., Soerjomataram, I., Dikshit, R., Eser, S., Mathers, C., Rebelo, M., Parkin, D.M.,

224 Forman, D., and Bray, F. 2015. Cancer incidence and mortality worldwide: sources, methods and 225 major patterns in GLOBOCAN 2012. International Journal of Cancer 136:E359-E386.

226 10.1002/ijc. 29210

227 Galon, J., Costes, A., Sanchez-Cabo, F., Kirilovsky, A., Mlecnik, B., Lagorce-Pages, C., Tosolini, 228 M., Camus, M., Berger, A., Wind, P., Zinzindohoue, F., Bruneval, P., Cugnenc, P.H., Trajanoski, 229 Z., Fridman, W.H., and Pages, F. 2006. Type, density, and location of immune cells within 230 human colorectal tumors predict clinical outcome. Science 313:1960-1964. 231 10.1126/science.1129139

232 Hwang, W.T., Adams, S.F., Tahirovic, E., Hagemann, I.S., and Coukos, G. 2012. Prognostic 233 significance of tumor-infiltrating $\mathrm{T}$ cells in ovarian cancer: a meta-analysis. Gynecologic 234 Oncology 124:192-198. 10.1016/j.ygyno.2011.09.039

235 Kaufman, D.S., Shipley, W.U., and Feldman, A.S. 2009. Bladder cancer. Lancet 374:239-249. 236 10.1016/S0140-6736(09)60491-8

237 Laghi, L., Bianchi, P., Miranda, E., Balladore, E., Pacetti, V., Grizzi, F., Allavena, P., Torri, V., 238 Repici, A., Santoro, A., Mantovani, A., Roncalli, M., and Malesci, A. 2009. CD3+ cells at the 239 invasive margin of deeply invading (pT3-T4) colorectal cancer and risk of post-surgical 240 metastasis: a longitudinal study. Lancet Oncology 10:877-884. 10.1016/S1470-2045(09)70186-X 241 Mahmoud, S.M., Paish, E.C., Powe, D.G., Macmillan, R.D., Grainge, M.J., Lee, A.H., Ellis, I.O., 242 and Green, A.R. 2011. Tumor-infiltrating CD8+ lymphocytes predict clinical outcome in breast cancer. Journal of Clinical Oncology 29:1949-1955 10.1200/JCO.2010.30.5037

Morales, A., Eidinger, D., and Bruce, A.W. 1976. Intracavitary Bacillus Calmette-Guerin in the treatment of superficial bladder tumors. J Urol 116:180-183. Kirilovsky, A., Nilsson, M., Damotte, D., Meatchi, T., Bruneval, P., Cugnenc, P.H., Trajanoski, Z., Fridman, W.H., and Galon, J. 2005. Effector memory T cells, early metastasis, and survival in colorectal cancer. $N$ Engl J Med 353:2654-2666. 10.1056/NEJMoa051424 Powles, T., O'Donnell, P.H., Massard, C., Arkenau, H.T., Friedlander, T.W., Hoimes, C.J., Lee, J.L., Ong, M., Sridhar, S.S., Vogelzang, N.J., Fishman, M.N., Zhang, J., Srinivas, S., Parikh, J., Antal, J., Jin, X., Gupta, A.K., Ben, Y., and Hahn, N.M. 2017. Efficacy and Safety of Durvalumab in Locally Advanced or Metastatic Urothelial Carcinoma: Updated Results From a Phase 1/2 Open-label Study. JAMA Oncol:e172411. 10.1001/jamaoncol.2017.2411 Sanmamed, M.F., and Chen, L. 2014. Inducible expression of B7-H1 (PD-L1) and its selective role in tumor site immune modulation. Cancer Journal 20:256-261. 10.1097/PPO.0000000000000061 
262 Siegel, R.L., Miller, K.D., and Jemal, A. 2017. Cancer Statistics, 2017. CA Cancer J Clin 67:7263 30. 10.3322/caac. 21387

264 Torre, L.A., Bray, F., Siegel, R.L., Ferlay, J., Lortet-Tieulent, J., and Jemal, A. 2015. Global 265 cancer statistics, 2012. CA Cancer J Clin 65:87-108. 10.3322/caac.21262

266 Tumeh, P.C., Harview, C.L., Yearley, J.H., Shintaku, I.P., Taylor, E.J., Robert, L., Chmielowski, 267 B., Spasic, M., Henry, G., Ciobanu, V., West, A.N., Carmona, M., Kivork, C., Seja, E., Cherry, 268 G., Gutierrez, A.J., Grogan, T.R., Mateus, C., Tomasic, G., Glaspy, J.A., Emerson, R.O., Robins, 269 H., Pierce, R.H., Elashoff, D.A., Robert, C., and Ribas, A. 2014. PD-1 blockade induces 270 responses by inhibiting adaptive immune resistance. Nature 515:568-571. 10.1038/nature13954

271 Wang, W., Kryczek, I., Dostal, L., Lin, H., Tan, L., Zhao, L., Lu, F., Wei, S., Maj, T., Peng, D., 272 He, G., Vatan, L., Szeliga, W., Kuick, R., Kotarski, J., Tarkowski, R., Dou, Y., Rattan, R., 273 Munkarah, A., Liu, J.R., and Zou, W. 2016. Effector T Cells Abrogate Stroma-Mediated 274 Chemoresistance in Ovarian Cancer. Cell 165:1092-1105. 10.1016/j.cell.2016.04.009

275 Zhang, L., Conejo-Garcia, J.R., Katsaros, D., Gimotty, P.A., Massobrio, M., Regnani, G., 276 Makrigiannakis, A., Gray, H., Schlienger, K., Liebman, M.N., Rubin, S.C., and Coukos, G. 2003. 277 Intratumoral $\mathrm{T}$ cells, recurrence, and survival in epithelial ovarian cancer. $N$ Engl J Med 278 348:203-213. 10.1056/NEJMoa020177 
Figure 1

Representative examples of CD8+ TILs immunostaining.

CD8+ TILs are distributed mainly at the interface of the tumor and tumor-adjacent stroma. A,

$B, C$ and $D(\times 100$ magnification) are representative images of scores of $0,1,2$, and 3 , respectively.

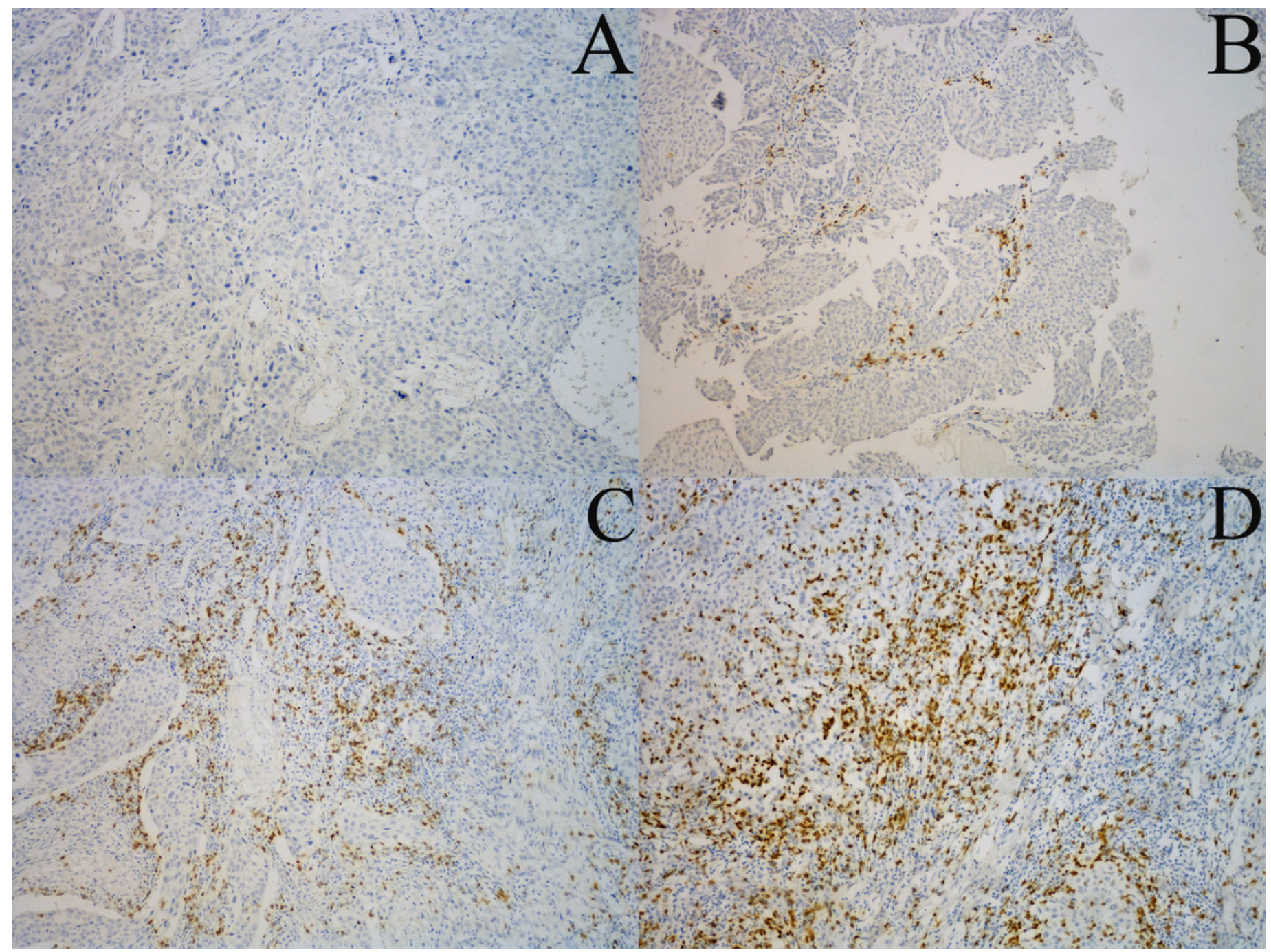


Figure 2

Kaplan-Meier survival curves with log-rank testing.

A. All UC of the bladder (CD8+, 90; CD8-, 36). B. Non-organ-confined UC of the bladder $(C D 8+, 35 ; C D 8-, 16)$. C. Organ-confined UC of the bladder (CD8+, 55; CD8-, 20).
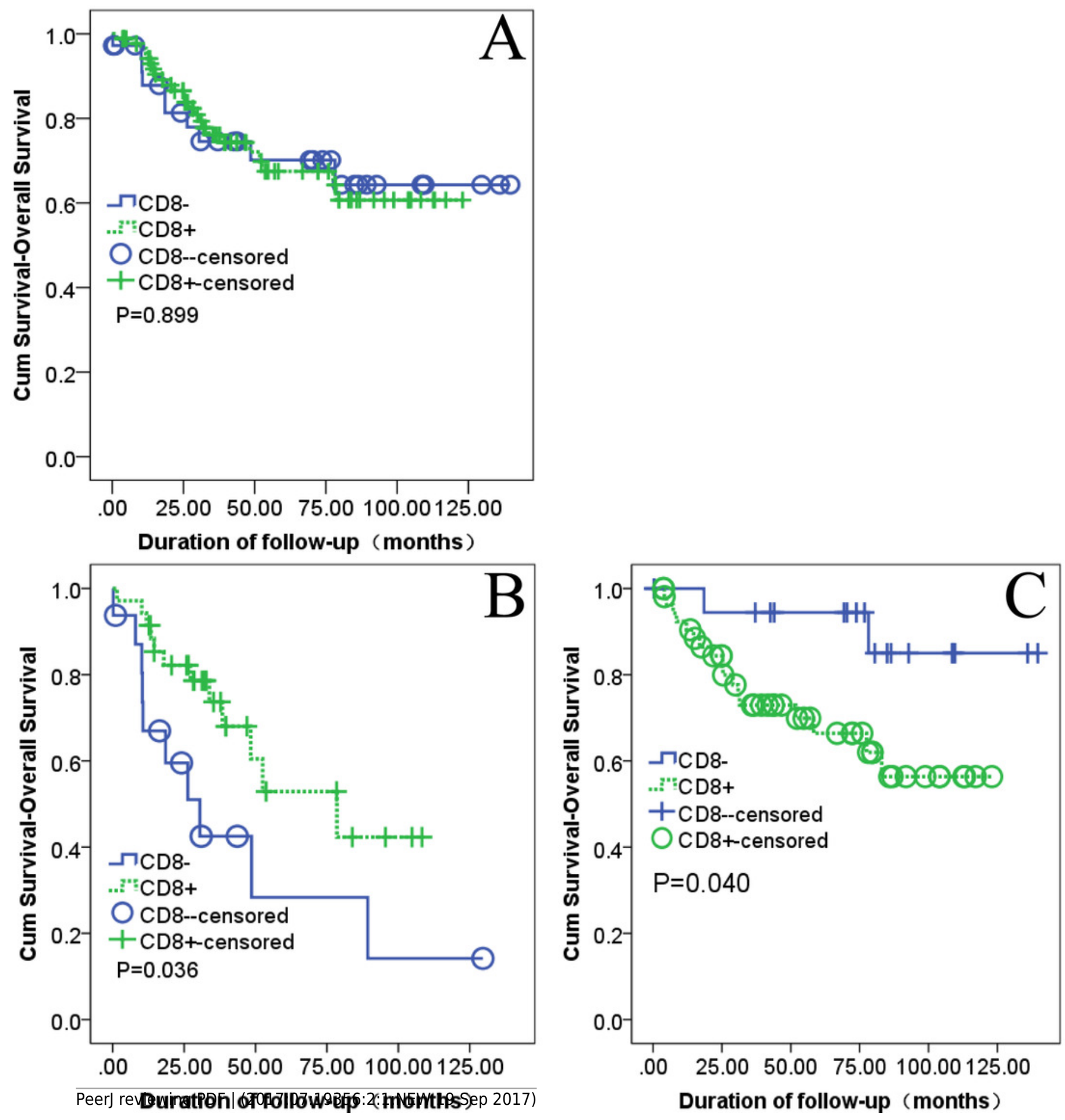
Table $\mathbf{1}$ (on next page)

Correlation between clinicopathological features and CD8+ TILS 


\begin{tabular}{|c|c|c|c|c|c|c|c|c|c|c|c|}
\hline \multirow[t]{2}{*}{ Characteristics } & \multirow[t]{2}{*}{$\mathrm{N}$} & \multicolumn{2}{|c|}{$\begin{array}{l}\text { Organ- } \\
\text { confined }\end{array}$} & \multicolumn{2}{|l|}{$\mathrm{P}$} & \multirow[t]{2}{*}{$\begin{array}{l}\text { Characteristic } \\
\text { s }\end{array}$} & \multirow[t]{2}{*}{$\mathrm{N}$} & \multicolumn{2}{|c|}{$\begin{array}{l}\text { Non-organ- } \\
\text { confined }\end{array}$} & \multicolumn{2}{|l|}{$\mathrm{P}$} \\
\hline & & CD8+ & $\begin{array}{l}\text { CD8 } \\
-\end{array}$ & $\begin{array}{l}\text { Fisher } \\
\text { test }\end{array}$ & exact & & & CD8+ & $\mathrm{CD} 8-$ & $\begin{array}{l}\text { Fisher } \\
\text { test }\end{array}$ & exact \\
\hline Age (years) & & & & 0.296 & & & & & & 0.760 & \\
\hline$<60$ & $\begin{array}{l}3 \\
6\end{array}$ & 24 & 12 & & & & $\begin{array}{l}1 \\
8\end{array}$ & 13 & 5 & & \\
\hline$\geq 60$ & $\begin{array}{l}3 \\
9\end{array}$ & 31 & 8 & & & & $\begin{array}{l}3 \\
3\end{array}$ & 22 & 11 & & \\
\hline Sex & & & & 1.000 & & & & & & 0.295 & \\
\hline Male & $\begin{array}{l}6 \\
4\end{array}$ & 47 & 17 & & & & $\begin{array}{l}4 \\
7\end{array}$ & 31 & 16 & & \\
\hline Female & $\begin{array}{l}1 \\
1\end{array}$ & 8 & 3 & & & & 4 & 4 & 0 & & \\
\hline pT stage & & & & 0.033 & & pT stage & & & & 0.345 & \\
\hline pTa-1 & $\begin{array}{l}3 \\
1\end{array}$ & 27 & 4 & & & pT3 & $\begin{array}{l}3 \\
4\end{array}$ & 25 & 9 & & \\
\hline pT2 & $\begin{array}{l}4 \\
4\end{array}$ & 28 & 16 & & & pT4 & $\begin{array}{l}1 \\
7\end{array}$ & 10 & 7 & & \\
\hline pN stage & & & & & & pN stage & & & & 0.746 & \\
\hline & & & & & & N- & $\begin{array}{l}3 \\
5\end{array}$ & 23 & 12 & & \\
\hline & & & & & & $\mathrm{N}+$ & $\begin{array}{l}1 \\
6\end{array}$ & 12 & 4 & & \\
\hline Nuclear Grade & & & & 0.107 & & & & & & & \\
\hline Low & $\begin{array}{l}2 \\
6\end{array}$ & 16 & 10 & & & & & & & & \\
\hline
\end{tabular}




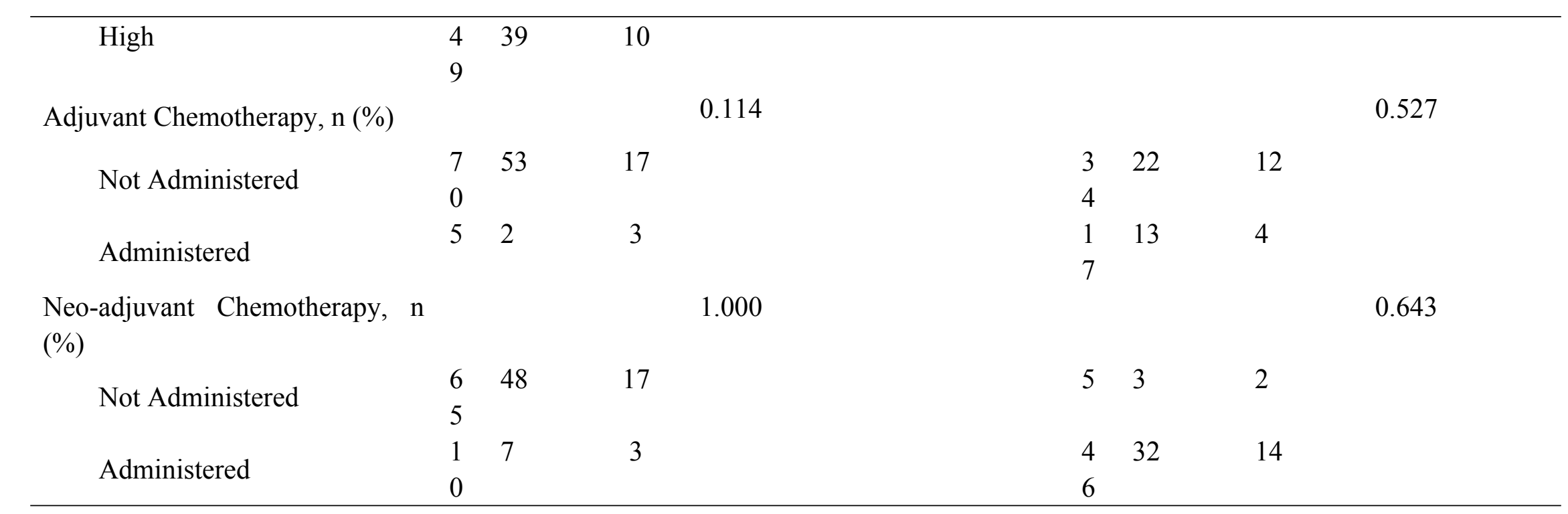


Table 2 (on next page)

Association between CD8 status and OS in the subset of 75 organ-confined and 51 nonorgan-confined urothelia carcinoma of the bladder patients in the Cox proportional hazards model 


\begin{tabular}{ccccccccc}
\hline & \multicolumn{2}{c}{ organ-confined } & & & \multicolumn{3}{c}{ non-organ-confined } & \\
\cline { 2 - 3 } \cline { 6 - 8 } & Risk ratio & $95 \% \mathrm{CI}$ & $\mathrm{P}$ & & Risk ratio & $95 \% \mathrm{CI}$ & $\mathrm{P}$ \\
\hline $\mathrm{CD} 8$-vs CD8+ & 0.212 & $0.048-0.932$ & 0.040 & & 2.397 & $1.031-5.573$ & 0.042 \\
\hline
\end{tabular}

1 Adjusted for age, sex, nuclear stage, T stage, $\mathrm{N}$ stage (only in non-organ-confined), neo-adjuvant

2 chemotherapy, and adjuvant chemotherapy. 